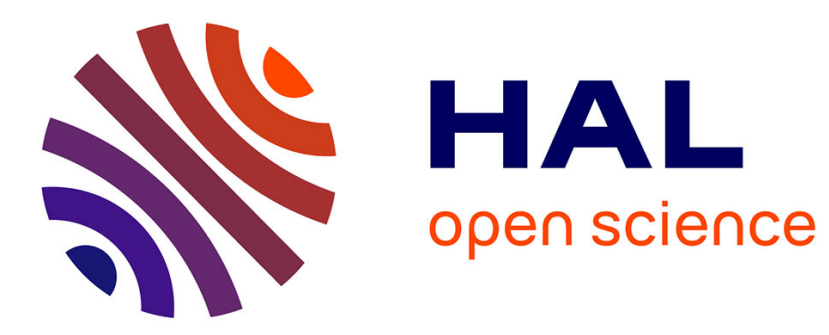

\title{
Winnicott sur les ondes pendant la Seconde Guerre mondiale : entre douleur et politique
}

Clara Lecadet

\section{To cite this version:}

Clara Lecadet. Winnicott sur les ondes pendant la Seconde Guerre mondiale : entre douleur et politique. Champ Psy, 2002, 28 (4), pp.69-84. 10.3917/cpsy.028.0069 . hal-01809374

\section{HAL Id: hal-01809374 \\ https://hal.science/hal-01809374}

Submitted on 6 Jun 2018

HAL is a multi-disciplinary open access archive for the deposit and dissemination of scientific research documents, whether they are published or not. The documents may come from teaching and research institutions in France or abroad, or from public or private research centers.
L'archive ouverte pluridisciplinaire HAL, est destinée au dépôt et à la diffusion de documents scientifiques de niveau recherche, publiés ou non, émanant des établissements d'enseignement et de recherche français ou étrangers, des laboratoires publics ou privés. 


\title{
WINNICOTT SUR LES ONDES PENDANT LA SECONDE GUERRE MONDIALE : ENTRE DOULEUR ET POLITIQUE
}

\author{
Clara Lecadet
}

L'Esprit du temps | Champ psychosomatique

\author{
$2002 / 4$ - no 28 \\ pages 69 à 84
}

ISSN 1266-5371

Article disponible en ligne à l'adresse:

http://www.cairn.info/revue-champ-psychosomatique-2002-4-page-69.htm

Pour citer cet article :

Lecadet Clara, «Winnicott sur les ondes pendant la Seconde Guerre mondiale : entre douleur et politique », Champ psychosomatique, 2002/4 no 28, p. 69-84. DOI : 10.3917/cpsy.028.0069

Distribution électronique Cairn.info pour L'Esprit du temps.

(c) L'Esprit du temps. Tous droits réservés pour tous pays.

La reproduction ou représentation de cet article, notamment par photocopie, n'est autorisée que dans les limites des conditions générales d'utilisation du site ou, le cas échéant, des conditions générales de la licence souscrite par votre établissement. Toute autre reproduction ou représentation, en tout ou partie, sous quelque forme et de quelque manière que ce soit, est interdite sauf accord préalable et écrit de l'éditeur, en dehors des cas prévus par la législation en vigueur en France. II est précisé que son stockage dans une base de données est également interdit. 


\title{
Winnicott sur les ondes pendant la Seconde Guerre mondiale : entre douleur et politique
}

\author{
Clara Lecadet
}

D’UNE CLINIQUE DE L'ÉVÉNEMENT...

L

ors d'une réception donnée en 1968 par son éditeur pour fêter le succès de la publication de ses interventions radiophoniques, Winnicott raconte que lorsqu'il s'est rendu en voiture à la BBC pour ses premières émissions, il roulait «parfois sur les décombres, mêlés de vitres brisées, laissés par les raids aériens de la nuit précédente ». Confronté aux destructions causées par la guerre et à la politique de l'évacuation, il fait de la radio l'instrument d'une tentative clinique inédite en s'adressant directement aux familles des enfants évacués et à leurs familles d'accueil. Winnicott a repéré, comme les hommes politiques et d'autres psychiatres de l'époque, l'importance de ce média en termes d'accès et d'impact sur les populations civiles.

À la différence de ses émissions d'après-guerre sur la petite enfance et sur les difficultés quotidiennes des mères dans l'éducation des enfants, ces premières émissions s'articulent autour d'un fait historique précis: la mise en œuvre du plan d'évacuation conçu par le gouvernement pour protéger les enfants des grandes villes du Royaume-Uni des bombardements aériens, et plus particulièrement de Londres qui était la plus directement menacée et fut la plus lourdement touchée. Quatre interventions radiophoniques, qui accompagnent au

Clara Lecadet, Psychologue clinicienne, Chargée de cours à 1'Université Paris V, 12 rue des Nonnains d'Hyères, 75004 Paris. 
début et à la fin de la guerre le plan dans ses différentes phases, esquissent une clinique de l'événement.

A partir du 1er septembre 1939, en quelques jours, environ 800000 enfants partent en train, accompagnés d'enseignants, pour les campagnes anglaises, vers des destinations pour la plupart du temps inconnues d'eux-mêmes et de leurs familles. La première émission suit la première grande vague d'évacuation, qui fut la plus spectaculaire et la plus marquante. Winnicott exprime l'ambivalence et l'intensité des sentiments éprouvés par les parents, désireux de protéger leurs enfants mais aussi déchirés par leur départ, et insiste tout particulièrement sur la douleur des mères.

En 1945, dans une seconde émission, Winnicott s'adresse aux familles qui ont accueilli les enfants pendant la guerre; il exprime la dette contractée par la société vis-à-vis d'elles et reconnaît la difficulté de la tâche qui leur a été assignée. Il effectue en même temps un rappel des souffrances des enfants durant ces années vécues dans un autre foyer.

En 1945, Winnicott s'adresse à nouveau aux parents des enfants évacués. Chapitre final de l'évacuation, ces deux émissions marquent la fin de la guerre et le retour des enfants dans leur famille d'origine.

Winnicott isole les grands moments de l'évacuation et, réservant à chacun des publics concernés par cette initiative une émission et un discours particuliers, il propose une analyse psychosociologique du choc de la séparation, de la douleur de l'absence et de la difficulté des retrouvailles. La tentative clinique ainsi mise en œuvre se présente comme une prise en charge collective, globale, de l'impact d'un événement tel que l'évacuation, dans laquelle le réconfort doit émaner de la reconnaissance publique des douleurs des familles.

\section{... À LA GESTION DE L'OPINION PUBLIQUE?}

Il existe un très grand nombre de témoignages d'anciens enfants évacués sur cette période ; certains font état de mauvais traitements subis dans les familles d'accueil, tous mettent en avant la rupture considérable que l'évacuation a représentée dans l'existence d'enfants citadins, souvent issus de milieux modestes, ayant fait pendant plusieurs années l'expérience d'un mode de vie différent et d'une famille qui, au départ, 
n'était pas la leur. Si tous ne sont pas directement critiques sur le principe même de l'évacuation, tous montrent l'intensité de ces bouleversements.

Par ailleurs, des études historiques récentes (Stuart Hylton, Their darkest hour ; the hidden history of the home front 19391945) tendent à assimiler l'organisation de ce plan à une vaste entreprise de propagande; les risques de bombardements de la part de l'aviation allemande auraient été largement surestimés et l'idée d'un plan d'évacuation sans précédent serait née dans l'esprit des gouvernants par crainte que les populations civiles, si elles étaient suffisamment bombardées, n'en arrivent à exiger la paix à n'importe quel prix.

Les détracteurs de ce plan ont à l'époque reproché aux autorités d'avoir fait pression sur les familles au moyen d'une campagne publicitaire: affiches montrant des enfants levant les yeux au ciel, l'air hagard et désespéré, avec en légende «Vous les mères, envoyez-les hors de Londres », exhortations radiophoniques sur la nécessité, voire les bienfaits de l'évacuation, comme le célèbre Children's hour de la princesse Elizabeth. Le gouvernement aurait ainsi obtenu le consentement d'une grande partie des familles, les autres, le plus souvent dans les milieux les plus aisés, ayant pris des dispositions personnelles pour mettre leurs enfants à l'abri. Si les premières évacuations passent relativement bien auprès de l'opinion (notamment la grande vague du $1^{\text {er }}$ au 3 septembre 1939), elles sont de plus en plus mal ressenties au fur et à mesure que la guerre avance. Un deuxième projet d'évacuation se solde en 1940 par un échec cinglant et l'impopularité à l'égard de ce système grandit.

Les critiques sur la légitimité du plan d'évacuation ainsi que l'usage nécessairement singulier pour un thérapeute d'un média de masse tel que la radio nous ont conduit à nous interroger sur le statut des interventions radiophoniques que Winnicott effectue pendant la guerre. On peut se demander en effet si la clinique, dans la version collective qui en est donnée au public de la BBC, n'a pas servi à discréditer la possibilité même d'un discours critique sur le plan d'évacuation. On peut se demander également où s'arrête la clinique et où commence la manipulation, les formes larvées de propagande, qu'elles soient ou non volontaires.

Quand il parle à la radio, Winnicott se situe au croisement du politique, du social et de la clinique, à une place qui inter- 
roge de façon assez exemplaire les rapports de la clinique et du politique. La guerre en donne un éclairage particulier, plus fort sans doute qu'à l'ordinaire.

Car, si les émissions sont entièrement centrées sur la douleur des familles et sur la mise en évidence des conflits psychiques inconscients induits par l'évacuation, en est exclue toute dimension polémique de critique ou de contestation du plan. La reconnaissance, le partage public de la douleur des familles apparaissent aussi comme des moyens d'appuyer la légitimité de la logique d'Etat. En d'autres termes, le travail de réconfort effectué par Winnicott sur les ondes est propre à fournir des conditions satisfaisantes à l'exécution du plan d'évacuation, dont Winnicott perçoit pourtant très tôt les imperfections, les limites et les risques.

L'engagement de Winnicott sur le front de l'évacuation est en effet pluriel. En dehors de la radio, il mène plusieurs actions, plus confidentielles, relatives à l'évacuation. Alors qu'il est psychiatre consultant au plan gouvernemental d'évacuation, il écrit plusieurs lettres pour alerter les autorités des risques liés à l'évacuation. Alors qu'il supervise des foyers créés pendant la guerre et gérés par des travailleurs sociaux pour les enfants difficiles qui n'ont pas pu être placés dans des familles d'accueil, il rédige des articles destinés aux enseignants et aux travailleurs sociaux qui s'occupent des enfants évacués.

\section{LA RECONNAISSANCE DE LA DOULEUR DES FAMILLES...}

Winnicott est amené à réfléchir pendant toute la durée de la guerre aux conséquences chez les jeunes enfants de la séparation prolongée induite par l'évacuation. Mais il y a une différence entre les développements théoriques sur les effets de la séparation et la manière dont il intervient dans la mise en œuvre du plan d'évacuation, l'ordre dans lequel il le fait et ceux à qui il s'adresse.

Il est ainsi intéressant que dans l'ordre des communications radiophoniques qu'il effectue de 1939 à 1945, ce soit d'abord aux parents et plus particulièrement aux mères qu'il s'adresse.

La tonalité que Winnicott donne à sa première intervention radiophonique au moment des premières évacuations est sans 
équivoque. Il parle de l'évacuation comme d'une «lourde épreuve » pour les parents, évoque les « appels au secours » de mères privées de leurs enfants et commence par ces mots cette émission toute entière destinée aux parents : «Les parents sont mieux placés que quiconque pour savoir ce qui convient aux enfants en matière de soins infantiles» (D.W. Winnicott, 1939).

Cette émission suit la première vague d'évacuation. Quelques centaines de milliers d'enfants ont été brutalement séparés de leurs parents, sans que ceux-ci aient eu la moindre information au moment du départ sur le lieu et la famille où séjournerait leur enfant. C'est dans ce contexte d'extrême émotion qu'il faut replacer cette première émission, dans laquelle Winnicott insiste sur l'intensité du choc émotionnel engendré dans toute la population par ces séparations à la fois inattendues, brusques, rapides, et, en termes de communication et de dialogue, très mal préparées par les autorités. Les critiques adressées au plan d'évacuation ont porté précisément sur le caractère secret de sa mise en place et sur l'absence de véritables consultations et concertations avec les autorités locales et avec les familles.

Il n'est certainement pas anodin que Winnicott commence cette première émission en réaffirmant le caractère fondateur de la fonction parentale et l'importance du savoir des parents au sujet de leurs enfants, qu' au moins symboliquement, le caractère purement étatique, dirigé, massif et peu personnalisé des évacuations a sérieusement mis à mal, et finisse son intervention en insistant sur l'indispensable reconnaissance de la douleur des familles. A ce titre, cette première émission apparaît comme un message de reconnaissance publique, à bien des égards politique, de la douleur des familles, qui passe ainsi du statut d'une expérience privée à celui d'une expérience collective, partagée. De là à faire de l'évacuation des enfants une contribution aux efforts et aux sacrifices exigés à la nation... il n'y a qu'un pas que Winnicott ne franchit justement pas!

Il y a selon lui quelque chose d'irréductible dans la douleur des familles que rien ne peut ni relativiser ni réellement justifier. Pour autant, il s'agit de rendre cette douleur supportable et cela passe semble-t-il par l'acceptation du plan, avec toutes ses conséquences.

«S'occuper des enfants est un travail parfois dur et 
exigeant, qui s'apparente à un effort de guerre. En revanche, être simplement privé de ses enfants représente un bien piètre effort de guerre qui ne peut en aucun cas tenter les parents et qui n'est supportable que dans la mesure où le côté douloureux en est reconnu.» (D.W. Winnicott, 1939).

\section{... ET L'APPUI IMPLICITE À LA LOGIQUE D'ETAT}

L'usage qu'il fait, dès lors, d'éléments empruntés à la théorie psychanalytique est ambigu, dans la mesure où ils semblent parfois mis au service du seul contexte de la guerre et des choix politiques du gouvernement. Ainsi de la minoration du désir des familles; Winnicott évoque certes abondamment la douleur des familles mais il ne cache pas que la prise en compte du seul désir des parents aboutirait à une impasse dans le contexte d'extrême urgence de la guerre, d'autant que le désir des parents est par définition souvent complexe, ambivalent. La sincérité des mères dans leur volonté de coopérer avec les autorités ne doit pas occulter, selon lui, l'existence de conflits inconscients, qui rendent cette coopération souvent problématique. Ces conflits se manifestent notamment dans l'ambivalence des relations à cet autre à qui l'on remet son enfant; jalousie, hostilité, soupçon de malnutrition et de maltraitance. Winnicott ne s'intéresse pas tant à la réalité des éventuels mauvais traitements subis par certains enfants dans leur famille d'accueil (ces mauvais traitements ne seront connus et dénoncés qu'après la guerre) qu'aux fantasmes des mères et des répercussions de ces fantasmes sur le comportement des enfants. Winnicott cite ainsi l'exemple des mères qui affichent ouvertement des sentiments hostiles à l'égard des nourrices; l'enfant se comporte mal afin de conforter sa mère dans l'idée qu'ailleurs, ce ne peut être que mal. A l'inverse, la surestimation ou la confiance aveugle dans la famille d'accueil peut amener les parents à ignorer, voire à adopter une attitude de déni vis-à-vis de la réalité vécue par l'enfant.

Quelle doit être l'attitude des autorités pour empêcher la prolifération des peurs, de fantasmes des parents? Il pense que le lien parents-enfants et la fonction parentale elle-même peuvent être maintenus par l'envoi aux parents de rapports rédigés sans triomphalisme, qui relatent la vie de l'enfant simplement, sans insister sur les éléments qui pourraient 
susciter chez les parents des sentiments excessifs, l'inquiétude ou bien encore la jalousie s'il apparaît que l'enfant vit trop bien dans sa nouvelle famille! Cette substitution de la bureaucratie à l'expression brute des sentiments des uns et des autres va dans le sens d'une volonté de «pacification» des affects affichée par Winnicott dans ses émissions.

Au-delà de la parole grave mais aussi soucieuse de réconfort du thérapeute, on peut voir dans cette première émission un discours qui articule subtilement l'affirmation implicite de la nécessité et de la légitimité du plan d'évacuation, et les aspects privés des tragédies provoquées par sa mise en œuvre. Est mise en avant l'objectivité du danger encouru par les enfants : "On demande aux mères vivant dans les villes de laisser partir leurs enfants, on le leur conseille, on les pousse à le faire. Elles ont souvent l'impression qu'on leur force la main ; elles ne réalisent pas que la brutalité de cette démarche est en réalité dictée par le danger des bombardements » (D.W. Winnicott, 1939).

Cette intervention tente de concilier le souci des familles et l'appui à la logique d'Etat: après la sidération première, doit venir le temps d'une coopération librement comprise et consentie; après le moment traumatique de la rupture les mères comprendront que l'Etat a agi ainsi non pour les destituer de leur fonction mais pour protéger efficacement les enfants; bref la réalité reprendra ses droits! Cette volonté d'apaisement et de conciliation de deux logiques contraires traduit assez bien la position de Winnicott pendant la guerre ; thérapeute engagé au titre de psychiatre consultant au plan gouvernemental d'évacuation, participant en cela à sa mise en place, et thérapeute soucieux de limiter les répercussions psychiques de l'évacuation sur les enfants et leurs parents.

Winnicott questionne l'organisation même de l'évacuation au regard de la douleur des mères et en même temps son message vise implicitement à faire passer auprès des familles le plan par la reconnaissance et la compréhension partagée de leur douleur.

On peut donc comprendre cette intervention comme le souci de diffuser un certain nombre de thèmes psychanalytiques susceptibles d'intéresser et d'aider les familles mais aussi de rendre possible l'évacuation en contenant leur angoisse et parfois leur colère. Situées à l'interface du psychologique et du politique, les émissions de la BBC jouent un rôle 
de contenant social ; elles traduisent l'intuition que Winnicott a du caractère profondément contenant de la reconnaissance de l'angoisse de chacun.

La position de Winnicott est complexe puisqu'elle se situe au croisement de logiques qui peinent à converger; alerter les pouvoirs publics en les interpellant sans détours sur les risques de l'évacuation et infléchir ainsi une politique d'évacuation mal pensée, parler directement aux familles par le biais de la radio dans le cadre d'un travail clinique sociétal, tout en effectuant un travail clinique plus traditionnel auprès des enfants déplacés.

Ce qui nous semble le plus intéressant, c'est la façon dont Winnicott construit du discours en fonction de ses interlocuteurs. Les émissions radiophoniques pour les familles au début de la guerre et les familles d'accueil à la fin de la guerre réalisent un équilibre quasi parfait entre la prise en compte de la logique d'Etat et la reconnaissance des douleurs personnelles. Néanmoins, si l'on s'éloigne des ondes et du grand public, le discours de Winnicott sur l'évacuation laisse entendre quelques dissonances.

\section{EN MARGE DE LA RADIO, LA CRITIQUE}

Les critiques les plus ouvertes sur le plan d'évacuation se développent en marge des émissions radiophoniques. Deux exemples vont dans ce sens. Dès décembre 1939, dans une lettre adressée au British Journal, Winnicott met en garde les pouvoirs publics contre les risques liés à l'évacuation des toutpetits; des séparations précoces risquent de favoriser de profonds remaniements de leur personnalité et l'émergence de comportements délinquants. Dans le sillage des travaux de Bowlby, antérieurs à la guerre, sur la séparation mère-enfant, il développe l'idée que, dans sa prime enfance, seule la présence de sa mère est susceptible d'assurer à l'enfant le sentiment de sécurité interne dont il a besoin pour se construire. A ce stade, la présence ou non de danger réel compte peu. Dans une émission de 1960, il revient sur l'enseignement qu'il a tiré de l'expérience de la guerre en ces termes: «Lors des raids aériens, les bébés n'avaient pas peur des bombes. En revanche, ils étaient affectés dès que leurs mères se mettaient à paniquer» (D.W. Winnicott, 1960). Winnicott anticipe ainsi, 
dès le début de la guerre, dans cette lettre au British Journal, les risques majeurs liés à l'application du plan d'évacuation aux très jeunes enfants. Le facteur pathogène ne consiste pas tant dans les bombardements que dans les séparations impliquées par l'évacuation.

De la même façon, Winnicott prend nettement ses distances vis-à-vis de l'évacuation dans le compte-rendu paru en 1941 dans New era d'une enquête systématique effectuée la même année au sujet de l'évacuation, The Cambridge evacuation survey: a wartime study in social welfare and education. Il déplore le manque de préparation psychologique des médecins aux conséquences de l'évacuation (trop concentrés selon lui sur les seuls maux du corps) et le fait que les enseignants aient été seuls chargés de l'évacuation des enfants (tâche qu'ils ont néanmoins fort bien accomplie). Il a des mots très durs sur le principe même de l'évacuation, qui contrastent avec le ton général de ses émissions sur la BBC: «L'évacuation était inévitable. On a souvent tenté à tort de minimiser les malheurs inhérents à l'exil et prétendu que l'évacuation est une bonne chose, une chose sensée. Il a pourtant fallu une guerre pour en faire l'expérience. Je pense que l'évacuation ne peut entraîner que des tragédies : soit les enfants sont perturbés sur le plan affectif et ne s'en remettront peut-être jamais complètement, soit ils sont heureux et ce sont les parents qui souffrent, convaincus que plus personne n'a besoin d'eux, pas même leurs propres enfants. A mon sens, le seul point positif de l'évacuation est qu'elle pourrait échouer.» (D.W. Winnicott, 1941).

Entre l'inquiétude du début de la guerre sur le sort des jeunes enfants et la condamnation lourde du principe même de l'évacuation dans ce compte-rendu de 1941, un grand fossé s'est creusé. Cette évolution du jugement de Winnicott sur l'évacuation n'apparaît pas dans les émissions de la BBC. En 1941, soit deux ans après les premières évacuations, il est tout à fait désabusé sur le bilan général de l'évacuation, même s'il continue à penser qu'elle était inévitable. Au même moment, à la suite du torpillage par la marine allemande de navires transportant des enfants évacués à l'étranger, l'évacuation atteint des sommets d'impopularité.

Winnicott ne laisse rien percer de ce désespoir sur le bilan de l'évacuation dans ses émissions de la fin de la guerre. Il n'y est, en effet, nullement question de «l'échec de l'évacuation», que Winnicott évoque, en 1968, dans une lettre à Robert Tod. 
Là encore, l'approche clinique a pris le pas sur toute évocation, toute référence à la politique menée par le gouvernement pendant la guerre. D'une grande solennité, les émissions sur le retour des enfants portent l'empreinte tragique des années que vient de traverser l'Angleterre. Mais elles ne s'y cantonnent pas. Dans certains cas, note Winnicott, l'évacuation aura été une expérience positive.

Il émet l'idée selon laquelle il faut que tout cela serve à quelque chose, notamment en matière d'éducation des enfants. Il souhaite que s'effectue une sorte de transmission de savoir entre les familles qui ont accueilli les enfants pendant la guerre et les familles qui en temps de paix vont être amenées à s'occuper d'enfants difficiles. Face au gâchis, à la tragédie, à un sentiment de désespoir très sensible dans ses textes de guerre, Winnicott semble dans ces dernières émissions qui marquent la fin de la guerre, combattre l'idée que tout cela aura été en vain.

Il se concentre sur les aspects pratiques de la problématique du retour, dont l'approche globale est rendue difficile par l'hétérogénéité des situations. En ce sens, il est beaucoup moins dans la généralisation que lors de ses premières émissions; il a une démarche plus proche de celle de ses émissions radiophoniques ultérieures, dans lesquelles il s'appuie sur de nombreux exemples et essaie de répondre aux problèmes un par un.

Winnicott effectue entre le début et la fin de la guerre un travail d'articulation intéressant entre le fait même de l'évacuation, ses difficultés, et le retour des enfants dans les familles. Historiquement, il est sans doute utile de préciser que beaucoup d'enfants sont rentrés dans leurs familles avant la fin de la guerre, et que ni l'évacuation ni le retour des enfants n'ont été des phénomènes homogènes. Il donne une égale importance dans ses émissions radiophoniques à la douleur de la séparation et aux difficultés des retrouvailles. Ce thème de la difficulté du retour et de la nécessité d'une phase de réadaptation de la part des enfants et de leurs familles après une longue période de séparation, il l'anticipe d'ailleurs dès sa première intervention. 


\section{ENJEUX}

Par l'usage de la radio et par les écrits concernant l'évacuation des enfants, Winnicott pose les jalons d'une vision de la clinique qu'il ne cessera de défendre et de développer par la suite; commencer par s'adresser aux parents lorsqu'il est question des enfants, s'adresser aussi à ceux qui, par leur métier, sont amenés à apporter une aide psychologique aux enfants et à leurs parents sans pour autant être forcément psychologues, pouvoir apporter aux familles un «service thérapeutique minimum ». Si nous avons insisté sur le fait que dans ses premières émissions Winnicott s'adresse d'abord aux parents et notamment aux mères des enfants évacués, c'est pour souligner qu'il met ainsi intuitivement en œuvre l'idée selon laquelle aucune transformation réelle de la vie de l'enfant ne peut être effective sans la médiation des parents. Cela peut sembler paradoxal au moment même où les éloignements, les distances imposées par l'évacuation sont bien réelles; mais Winnicott, par la mise en évidence des conflits inconscients qui accompagnent ces séparations, cherche à montrer au contraire que les représentations que se font les parents de l'absence de leur enfant continuent à conditionner pour une part le comportement de celui-ci.

Par ailleurs, dans cette vision de la clinique qui s'esquisse avec la diffusion de ces émissions, et cela est sans doute encore renforcé par l'urgence de la guerre, le thérapeute apparaît comme celui qui peut, par les médias notamment, assurer ce minimum de conseils et d'information théorique dont les parents ont besoin «Dans ma clinique, ma devise est : quel est le minimum indispensable?».

Les émissions et les écrits sur l'évacuation ouvrent également un vaste champ de réflexion sur les rôles que doivent respectivement tenir les parents et l'Etat dans l'éducation des enfants ; une réflexion assez innovante sur leurs compétences respectives, leurs champs d'action, leur autonomie et la question de la légitimité de l'Etat d'intervenir ou non dans la sphère familiale. Cette question, on la retrouve comme une constante dans tout le travail ultérieur de Winnicott sur le placement des enfants difficiles; elle est toujours présentée comme un problème.

Cette réflexion s'accompagne d'une pensée du politique 
qui se déploie chez Winnicott en particulier au moment de la guerre sur le sens de la démocratie, sur le rôle des gouvernants ou sur le thème de la liberté. Winnicott insiste sur le fait que, dans une démocratie, l'Etat ne peut user d'aucun argument, aussi légitime fut-il, pour se substituer entièrement aux parents. Il est par conséquent essentiel que le caractère volontaire de l'évacuation ait été affirmé, même si Winnicott n'ignore pas que ce consentement a été, dans de nombreuses familles, arraché et que les pressions gouvernementales ont donné à de nombreuses familles le sentiment qu'on leur avait volé leur décision.

On a l'impression que, sans jamais être formulée vraiment, la question qui circule en filigrane dans les écrits et les interventions de Winnicott pendant la guerre, est de savoir s'il n'y avait finalement pas plus à perdre, du point de vue de la démocratie et de la santé psychique des enfants, en intervenant aussi massivement dans l'organisation des familles qu'en respectant leur intégrité. Ce dilemme terrible, probablement impossible à trancher, apparaît comme le point d'achoppement de ses réflexions et de son expérience pendant la guerre.

\section{L'EXPÉRIENCE INSTITUTIONNELLE DE WINNICOTT PENDANT LA GUERRE ET LA PROBLÉMATIQUE DE LA DÉLINQUANCE}

Aux problèmes pratiques et ponctuels posés par l'évacuation des enfants, auxquels Winnicott tente de répondre par différents types d'initiatives (interpeller les pouvoirs publics, réconforter les familles par une communication accrue autour des questions psychologiques posées par l'évacuation) s'associe une réflexion sur l'étiologie des comportements délinquants et sur le lien entre séparation et délinquance.

Cette réflexion se trouve enrichie par de nouvelles pratiques, puisque les autorités découvrent, à l'occasion de la mise en œuvre du plan d'évacuation, l'existence d'un certain nombre d'enfants réfractaires au placement prévu dans les familles d'accueil. Leur prise en charge va dès lors être un objet de préoccupation pour les autorités, les travailleurs sociaux et les médecins.

Il convient en effet de distinguer deux aspects de la mise en œuvre du plan d'évacuation: 
- l'évacuation des enfants issus de foyers stables vers des familles d'accueil habitant en dehors des grandes villes - ce sont principalement aux parents de ces enfants et aux familles d'accueil que Winnicott s'adresse à la radio -;

- l'évacuation d'enfants réputés difficiles, qui, déjà avant la guerre, ne bénéficiaient pas d'un environnement stable. Des foyers sont créés pour ces enfants qui ne peuvent être placés en famille d'accueil ; ce sont des petites structures dont l'activité est centrée sur les soins, au sens large, où l'entend Winnicott (comme le suggère le verbe anglais to care: se soucier de, prendre soin, se préoccuper et s'occuper de quelqu'un). Cette définition du soin excède le seul domaine des soins médicaux; c'est par exemple dans la régularité des repas et des activités, c'est-à-dire dans la structuration même du temps, et dans la prise en charge personnalisée des enfants que réside la dimension thérapeutique de ces centres. Winnicott s'appuie sur l'expérience de ces foyers thérapeutiques pour tenter, après la guerre, d'articuler le fait de la séparation et le sentiment de déprivation qu'elle entraîne, avec l'apparition de la tendance antisociale, qui est une notion plus générale, moins connotée socialement que la notion de délinquance.

Dans ses émissions sur la BBC, il insiste sur la dimension antisociale des réactions que les enfants manifestent à la perte de leur environnement et sur le caractère positif de ces réactions. Dans un article de 1967, il reprend le thème de la délinquance comme signe d'espoir. Dans ses textes sur le traitement des délinquants ou dans certaines interventions radiophoniques ultérieures sur le sentiment de sécurité du nourrisson, Winnicott se réfère à plusieurs reprises à l'expérience de la guerre. Elle lui a permis, semble-t-il, de développer l'hypothèse selon laquelle n'importe quel enfant peut être amené à avoir un comportement antisocial quand on le prive brusquement de son environnement habituel, et pas seulement les enfants réputés difficiles : énurésie, apathie, vols, provocations verbales à l'égard des adultes, etc. Dès lors, la dimension antisociale du comportement concerne tous les enfants et plus seulement ceux qui sont stigmatisés comme «délinquants». C'est une manifestation commune.

Il s'est ainsi produit un apport en retour de l'expérience de terrain avec des enfants difficiles à la compréhension du développement psychoaffectif des enfants dans leur rapport à leur environnement. 
En outre, le plan d'évacuation a permis la mise en place d'un cadre institutionnel pérenne en matière de soins et de prévention pour les adolescents ayant des conduites antisociales. Winnicott souligne, dans plusieurs écrits, l'importance du rôle de l'institution dans la prise en charge thérapeutique de ces adolescents ainsi que l'ambiguïté de la problématique antisociale, qui «force» la psychanalyse à adopter une posture à la fois théorique et pratique. Winnicott décrit les limites d'une approche purement psychanalytique dans le traitement des enfants antisociaux.

Ce sont donc des enfants échappant à la grille des placements en famille d'accueil prévue par le plan d'évacuation qui ont suscité l'aménagement de structures propres à les accueillir. La guerre a rendu ces enfants plus visibles et leur prise en charge plus urgente. Et cela, comme l'explique Winnicott dans un article de 1947, rédigé avec Clare Britton («Le placement des enfants difficiles peut-il être thérapeutique ?»), ne correspondait ni à un élan charitable ou humaniste de la part des autorités, mais plutôt au souci de répondre à la très forte pression sociale d'une opinion qui redoutait de voir les campagnes anglaises sillonnées par des gamins errants commettant vols et larcins.

Winnicott retient de ce temps de guerre une leçon qu'il voudrait voir appliquée en temps de paix ; les autorités ont en effet préféré pendant la guerre recourir aux soins plutôt qu'aux tribunaux, en laissant, qui plus est, une large part aux initiatives des travailleurs sociaux concernant l'aménagement de nouveaux lieux, souvent même en les encourageant. La part belle laissée pendant la guerre aux initiatives locales pour la prise en charge des enfants difficiles impose, selon Winnicott, de réfléchir aux raisons pour lesquelles, en temps de paix, la préférence des pouvoirs publics va généralement à la mise en place de politiques répressives.

\section{REMARQUE FINALE}

Dans la littérature relative au travail et à l'expérience de Winnicott, la guerre est souvent présentée comme un moment charnière mais sans que soit précisée la complexité de son positionnement par rapport à l'évacuation des enfants. Dans cette notion de moment charnière, on croit parfois entendre 
«moment magique», comme si la guerre était le «sésame ouvre-toi » de toutes les questions laissées en suspens en temps de paix. Non seulement une telle vue de l'esprit est moralement contestable mais elle semble fausse si on examine de près la position nécessairement complexe, tendue, des thérapeutes en temps de guerre. Il nous semble en effet que c'est plutôt tout l'impensé de la guerre, les dilemmes insolubles, les tensions qu'elle suscite dans les pratiques et dans l'engagement luimême, qui peuvent être éventuellement un moteur pour des réflexions ultérieures. D'ailleurs, si la référence à son expérience de la guerre en tant que thérapeute est récurrente dans l'œuvre de Winnicott, elle demeure précisément une pure référence et Winnicott ne prend jamais le risque de son instrumentalisation.

\section{BIBLIOGRAPHIE}

BRITTON C., WINNICOTT D.W. (1947). Le placement des enfants difficiles peut-il être thérapeutique?, (1984). Déprivation et délinquance. Paris : Payot, 1994, 73-90.

WINNICOTT D.W. (1939). Les mères déprivées, (1984). Déprivation et délinquance. Paris : Payot, 1994, 46-54.

BOWLBY J., MILLER E., WINNICOTT D.W. (1939). Lettre au British Medical Journal, (1984). Déprivation et délinquance. Paris : Payot, 1994, 25-27.

WINNICOTT D.W. (1940). A propos des objectifs de la guerre, (1986). Conversations ordinaires. Paris: Gallimard, 2000, 239-251.

WINNICOTT D.W. (1941). L'évacuation dans la région de Cambridge: problèmes sociaux et éducatifs, (1984). Déprivation et délinquance. Paris : Payot, 1994, 35-38.

NORTH R., WINNICOTT D.W. (1944). Correspondance avec un magistrat, (1984). Déprivation et délinquance. Paris : Payot, 1994, 195-199.

WINNICOTT D.W. (1945). Les enfants évacués, (1984). Déprivation et délinquance. Paris: Payot, 1994, 55-60.

WINNICOTT D.W. (1945). Le retour des enfants évacués, (1984). Déprivation et délinquance. Paris: Payot, 1994, 61-66.

WINNICOTT D.W. (1945). Les enfants sont rentrés, (1984). Déprivation et délinquance. Paris : Payot, 1994, 67-72.

WINNICOTT D.W. (1948). Les foyers d'accueil en temps de guerre et en temps de paix, (1984). Déprivation et délinquance. Paris : Payot, 1994, 91-96.

WINNICOTT D.W. (1950). Quelques réflexions sur le sens du mot « démocratie», (1986). Conversations ordinaires. Paris: Gallimard, 2000, 273-294.

WINNICOTT D.W. (1960). Faut-il dire «non»?, (1993). Conseils aux 
parents. Paris : Payot, 1995, 43-67.

WINNICOTT D.W. (1967). La délinquance, signe d'espoir, (1986). Conversations ordinaires. Paris : Gallimard, 2000, 99-109.

\section{RÉSUMÉ}

Si les émissions radiophoniques effectuées par Winnicott pendant la seconde guerre mondiale au sujet de l'évaluation des enfants ont une fonction de «contenant social » par rapport à l'angoisse et à la douleur des familles et soutiennent implicitement les choix gouvernementaux en matière d'évacuation, Winnicott réserve ses critiques et sa condamnation lourde du principe même de l'évacuation à des écrits confidentiels.

Ce fossé entre des prises de position apparemment contradictoires traduit la complexité et la diversité des engagements tenus par Winnicott pendant la guerre, à l'origine de ses réflexions sur le rôle de l'Etat dans la prise en charge des enfants difficiles et sur les conditions d'émergence de la tendance antisociale.

Mots-clés : Clinique de l'événement - Clinique sociétale - Déprivation précoce - Tendance antisociale - Délinquance.

\section{SUMMARY}

While the radio programs broadcast by Winnicott during the Second World War about the evacuation of children serve as «social content» relative to the anguish and pain felt by families, and implicitly support the government's choices concerning the evacuation, Winnicott keeps his criticisms and his stern condemnation of the very principle of the evacuation for confidential writings.

This gap between apparently contradictory stands shows the complexity and diversity of the positions taken by Winnicott during the war, and they are at the origin of his reflexions on the role of the State in taking charge of problem children and on the conditions under which antisocial tendencies emerge.

Key-words : Clinic of the event - Societal clinic - Early deprivation Antisocial tendency - Deliquency. 